\title{
Combining image analyses tools for comprehensive characterization of root systems from soil-filled rhizobox phenotyping platforms**
}

\author{
Mouhannad Alsalem ${ }^{1}$, Aliyeh Salehi ${ }^{1,2}$, Jiangsan Zhao $^{3,4}$, Boris Rewald ${ }^{3}$, and Gernot Bodner ${ }^{1}$ (1) \\ ${ }^{1}$ Department of Crop Sciences, University of Natural Resources and Life Sciences, Konrad-Lorenz-Strasse 24, 3430 Tulln, Austria \\ ${ }^{2}$ Department of Sustainable Agricultural Systems, University of Natural Resources and Life Sciences, Gregor-Mendel-Straße 33, \\ 1180 Vienna, Austria \\ ${ }^{3}$ Department of Forest and Soil Sciences, University of Natural Resources and Life Sciences, Peter-Jordan-Straße 82, \\ 1190 Vienna, Austria \\ ${ }^{4}$ Institute for Sustainable Agro-ecosystem Services, University of Tokyo, 1 Chome-1-1 Midoricho, Nishitokyo, \\ Tokyo 188-0002, Japan
}

Received August 6, 2021; accepted October 15, 2021

\begin{abstract}
Root traits are fundamental for the resilience of plants under stress. Image-based phenotyping can provide relevant datasets to reveal the underlying root traits. However, root phenotyping is still hampered by methodological constrains, in particular the extraction of root traits from images taken under semi-natural conditions. In this study, we thus propose a strategy for analysing root images from rhizoboxes. Utilizing three Vicia faba genotypes and two soil moisture conditions, we applied software tools featuring distinctive types of root descriptors. We determined their accuracy in terms of root length measurement, inference from surface-visible root axes with regard to total root length, inter-relation between root architectural descriptors and their relevance to plant transpiration. Our results show that different image analysis tools provide similar root length estimates despite specific segmentation approaches. Several root architectural descriptors are also inter-comparable. Using structural equation modelling, we identified the relevant phenotyping root traits thereby characterizing root size and branching which drives plant transpiration. We conclude that rhizobox systems are a promising platform for root phenotyping. Future developments in image analysis should overcome the requirement for manual post-processing (e.g. gap closure) and automate root architecture measurement thereby improving throughput and thus the range of rhizobox phenotyping applicability for plant breeding.

Keywords: faba bean, image analysis, rhizobox, root phenotyping, root architecture, structural equation model
\end{abstract}

*Corresponding author e-mail: gernot.bodner@boku.ac.at

**This work was partially received funding from the European Union's Seventh Framework Program for research, technological development and demonstration under grant agreement No. 613781 (EUROLEGUME) (2014-2017).

\section{INTRODUCTION}

Functional plant traits have been recognized to be effective predictors of ecosystem function and plant growth strategies. In this context, root traits have gained significant attention in plant and soil research. Root traits such as root angle, specific root area, root diameter, root length density and total root length are essential for plant productivity, particularly under conditions of limited resource availability, and in turn influence the characteristics of the soil and ecosystem. In cropping systems, cultivars with site-adapted root systems are expected to enhance water and nutrient use efficiency, yield stability, and resilience during periods of climate change.

In the context of trait-based breeding, the integration of root systems, however, implies two major challenges: i) measurement/screening of large populations to rank candidates according to their performance, and ii) targeting those traits which are most promising for further selection and crossing. Root systems are complex organs of various sizes and structures resulting from spatial and temporal factors, cellular-level processes of elongation, branching and bending (Hodge et al., 2009). As a consequence, plant roots can also be characterized by parameters measured at different observation scales, from composite descriptors (e.g. total root length or shape of the root system, Kashiwagi

(C) 2021 Institute of Agrophysics, Polish Academy of Sciences 
et al., 2006, Freschet et al., 2021) to single traits (lateral branching number, emergence angle of laterals, Chen et al., 2017). In addition, the different types of root descriptors used in comparative root studies are related to different classification schemes which have emerged over the course of the entire history of root research (branching topology, geometrical shape, developmental order, see Freschet et al. (2021) for a recent review concerning root classification schemes and measurement protocols). Finally, the relationship between root traits and the agronomic/breeding target of improved drought resistance due to higher root water uptake is complex (Vadez, 2014). Thus, optimal measurement strategies for root systems would also consider the linkage between root descriptors and root functionality.

The number of root datasets has increased to a significant extent with the advancement of image-based phenotyping. Advanced methods for observing root systems non-destructively such as MRI imaging or X-ray tomography are expensive, require specialized equipment and are still limited in resolution at the fine root scale. Optical imaging approaches, using digital cameras or a scanner, are thus more frequently used to identify morphological, physiological, anatomical and biochemical traits. Rhizobox imaging is a root phenotyping approach which involves plants growing in soil-filled containers beyond the seedling/juvenile stages. This setup aims to approximate field-growing conditions (Nagel et al., 2012; Bodner et al., 2017). It may be considered to be an intermediate approach between root phenotyping on artificial media (filter paper, agar plates) and field root imaging with minirhizotrons (Johnson et al., 2001 ). The roots are only partially visible - about $20-30 \%$ (Pfeifer et al., 2014; Bodner et al., 2018) to $75-85 \%$ of the total root length (Bontpart et al., 2020). The complexity of the data sets is increased due to discontinuities within the visible root system structures (Chen et al., 2019) and the increasing overlap of the dense mature root systems (Kimura et al., 1999).

Image-based root phenotyping is expected to improve information concerning root system characteristics beyond classical field data (biomass, length, surface area per depth, specific root length/area, diameter). It provides an in-si$t u$ observation methods to track the development of root architecture over time (lateral numbers, angles, topology, Downie et al., 2015) as well as root-soil interactions influencing the shape of the root systems (inorganic nutrients, Wagner et al., 2020). These root architectural and functional traits are highly relevant to achieving better induction of potentials for improved stress resistance: e.g., a steep root angle essentially drives better access to subsoil water in dry ecosystems (Manschadi et al., 2006), while high lateral branching facilitates the superior exploitation of non-mobile nutrients such as phosphorous (Lynch, 2011). This is particularly important for legumes that have a comparatively high P demand (Pang et al., 2018), while - compared to the fibrous root system of monocots - the root density tends to be lower (Haling et al., 2016).
While image analysis has been identified as a general 'bottleneck' of current plant phenotyping (Minervini et al., 2015) which has led to many image analysis tools being developed over the last few decades (Lobet, 2017), this still holds particular true for root image analysis. For instance, Delory et al. (2017) compared different root length estimates from digital image analyses using the software packages WinRhizo ${ }^{\circledR}$ (Regent, Quebec, Canada) and ImageJ (NIH, USA) with the macro IJ_Rhizo (Pierret et al., 2013). They concluded that a comparison of root length measurements is substantially influenced by the software used due to the different underlying methods. Rose and Lobet (2019) compared IJ_Rhizo and WinRhizo with regard to the accuracy of the root diameter and volume estimates at different image resolutions. Similarly, they concluded that both software and image quality have a significant impact on reported root measurements. In recent times, approaches utilizing machine-learning have been presented to improve root segmentation and tracking and also to overcome some of the problems with soil-grown root images. Indeed, in-situ root images can be challenging for image analysis software due to i) the low contrast between root and soil in the case of colour-based segmentation (Wang et al., 2019), ii) the growth in parallel and/or high overlap of root axes when plants are grown over longer time periods (Himmelbauer $e t$ $a l ., 2004)$, and iii) the partial visibility of soil-grown roots at the imaged surface (Chen et al., 2019).

Therefore, the utility of root phenotyping critically depends on analytical strategies and adequate software tools to extract quantitative descriptors from root images that capture functional traits. In particular, an imaging and analytical strategy should be essentially based on the relationship between root traits and a specific target function, e.g. improved transpiration or the nutrient uptake of crops under resource limiting conditions (Vadez, 2014; Chen et al., 2018).

In this study, we propose a strategy for an affordable and comprehensive root image analysis of 2D RGB images that are typically obtained from rhizobox phenotyping platforms. In addition, we demonstrate the use of structural equation modelling (SEM) to identify key root traits related to water uptake/transpiration rates. We applied two software tools for the root image analysis of rhizobox-grown faba bean which provide (partially) distinctive types of root descriptors. Our aims were i) to determine the accuracy of root length measurement when directly applied to RGB as compared to manually tracked binary images, ii) test the correlation between surface-visible root axes of rhizobox systems and total root length, and iii) assess the nature of the inter-relation among root architectural descriptors and their role in capturing the architectural fundaments of overall root system size and functioning. From the results of this study, we expect to define a set of relevant root target traits 
with their related image processing/analyses requirements in order to maximize the usability of rhizobox systems for functional root phenotyping.

\section{MATERIALS AND METHODS}

A rhizobox experiment was conducted at the University of Natural Resources and Life Sciences, Vienna (BOKU) at UFT Tulln to investigate the root architecture and phenotyping of faba bean (Vicia faba L.). The experiment was implemented in a controlled growth chamber equipped with 8 LED-lamps providing homogeneous illumination $\left(450 \mu \mathrm{mol} \mathrm{m}^{-2} \mathrm{~s}^{-1}\right)$. The ambient conditions were set to a relative humidity at $45 \%$, with a temperature of $25^{\circ} \mathrm{C}$ during the day and $16^{\circ} \mathrm{C}$ at night, and the duration of illumination at $16 \mathrm{~h} \mathrm{light} / 8 \mathrm{~h}$ darkness.

A rhizobox phenotyping platform was used as a nondestructive method for imaging roots through a glass observation window. Detailed information concerning the rhizobox setup may be found in Bodner et al. (2017). The rhizoboxes $(30 \times 100 \times 1 \mathrm{~cm})$ were filled with field top soil (silt loam from a calcareous chernozem with total nitrogen and carbon contents of 0.19 and $3.94 \%$, respectively) and a bulk density of $1.3 \mathrm{~g} \mathrm{~cm}^{-3}$ corresponding to values typically found in agricultural soils. The soil was sieved to a particle size of $2 \mathrm{~mm}$, removing stones and large detrital materials, thereby allowing for homogeneous filling. The rhizoboxes were positioned at a $40^{\circ}$ angle to maximize the root visibility at the observation window.

The faba bean root data used for this study were obtained in an experiment evaluating three cultivars (Favel and FB 3293 from Portugal, Jõgeva from Estonia) using two water regimes. The seeds were inoculated with AMF spores (Funneliformis mosseae BEG95, Rhizophagus intraradices, and Funneliformis geosporum BEG199; Aleš Látr, Symbiom, Czech Republic) and a rhizobia suspension which was applied through dipping. The seeds were pre-germinated in darkness at $20^{\circ} \mathrm{C}$ in an incubator until the radicle emerged and grew to a length of about $2-3 \mathrm{~cm}$. Then one seedling was carefully placed in the middle of each Rhizobox at a depth of $3 \mathrm{~cm}$ using a screwdriver and tweezers. Apart from using three different cultivars, variation in root trait expression for the development of an image analysis strategy was obtained by varying the water regime (100 and 50\% plant-available water), the rhizoboxes were watered every 2 nd day. Every week, the rhizoboxes were weighed $( \pm 1 \mathrm{~g})$ and re-watered until they returned to their initial weight. Changes in individual rhizobox weights were used to calculate water loss over time. As the rhizoboxes were sealed with cling film to minimize soil evaporation and no drainage was recorded, the change in weight due to water loss was assumed to be equivalent to plant transpiration $\left(\mathrm{ml} d a y^{1}\right)$ and these values were added up to obtain the cumulative transpiration ( $\mathrm{ml})$. In total, 20 rhizoboxes were used in this study, producing three to four replicates per treatment ( 3 cultivars $\mathrm{x} 2$ watering regimes), each one was imaged at three development stages representing two principal growth stages, i.e. vegetative (BBCH 12 and 14 with two and four leaves unfolded respectively) and flowering (BBCH 60).

For imaging roots visible at the transparent surface, rhizoboxes were placed into an imaging box to shield them from ambient light (see Bodner et al., 2017 for details). Images were taken using a digital camera (EOS 6D; Canon Inc., Tokyo, Japan) with a resolution of $3648 \times 5472$ pixels at the top and bottom positions to cover the upper and the lower half of the rhizobox, respectively. The PC software Adobe Photoshop v. CS6 (Adobe, USA) was used to merge the images into one RGB raw image.

Figure 1 illustrates an example of image processing and the analysis pipeline used to obtain quantitative root system traits from the rhizobox images. In brief, unprocessed RGB images (Fig. 1A) were analysed using the commercial software WinRhizo Pro V.2013 (Regent, Quebec, Canada) based on colour segmentation (Fig. 1C). The processing of images via manual tracking and conversion into binary images (Fig. 1B) was performed in order to 1) assess the accuracy of RGB colour segmentation and 2) analyse the architectural traits requiring gap closure by user interaction. An analysis of the binary images was then conducted using both WinRhizo (Fig. 1D) as well as the open source software RootNav v.1.8.1 x64 (Pound et al., 2013, Fig. 1E). anual tracking was performed using a graphic tablet (Intuos Pro, Wacom Europe, Germany) and the PC software CorelDraw X7 (Corel Corporation, Canada).

After the final imaging at $\mathrm{BBCH} 60$, the roots were washed from soil (following Himmelbauer et al., 2004, two sieves of 2000 and $250 \mu \mathrm{m}$ were used to minimize fine root loss, the technique of floatation in water was applied to manually separate roots from organic debris), rinsed and scanned using a Perfection v700 scanner (with a transparency unit, 400 dpi, grey-scale, Epson, Japan). An analysis of total root length (including the non-surface visible roots) was conducted in order to assess if surface-visible roots are a meaningful approximation of whole plant root systems - using root length as a test trait which is obtained by all methodologies.

Beyond the different approaches to root segmentation and tracking, WinRhizo and RootNav provide different descriptors for a comprehensive characterization of root systems. Table 1 provides the respective image analysis parameters used here to characterize size (length, hull area, external path length) and branching pattern (lateral number, emergence angle, magnitude) of the root systems. The magnitude and external path length are descriptors from Fitter's classification based on segments (links) between branches in a root system (Fitter, 1987). The magnitude captures the intensity of the branching via external links ending in a tip, the external path length is a size related parameter describing the sum of all links from the root base to the external links. 


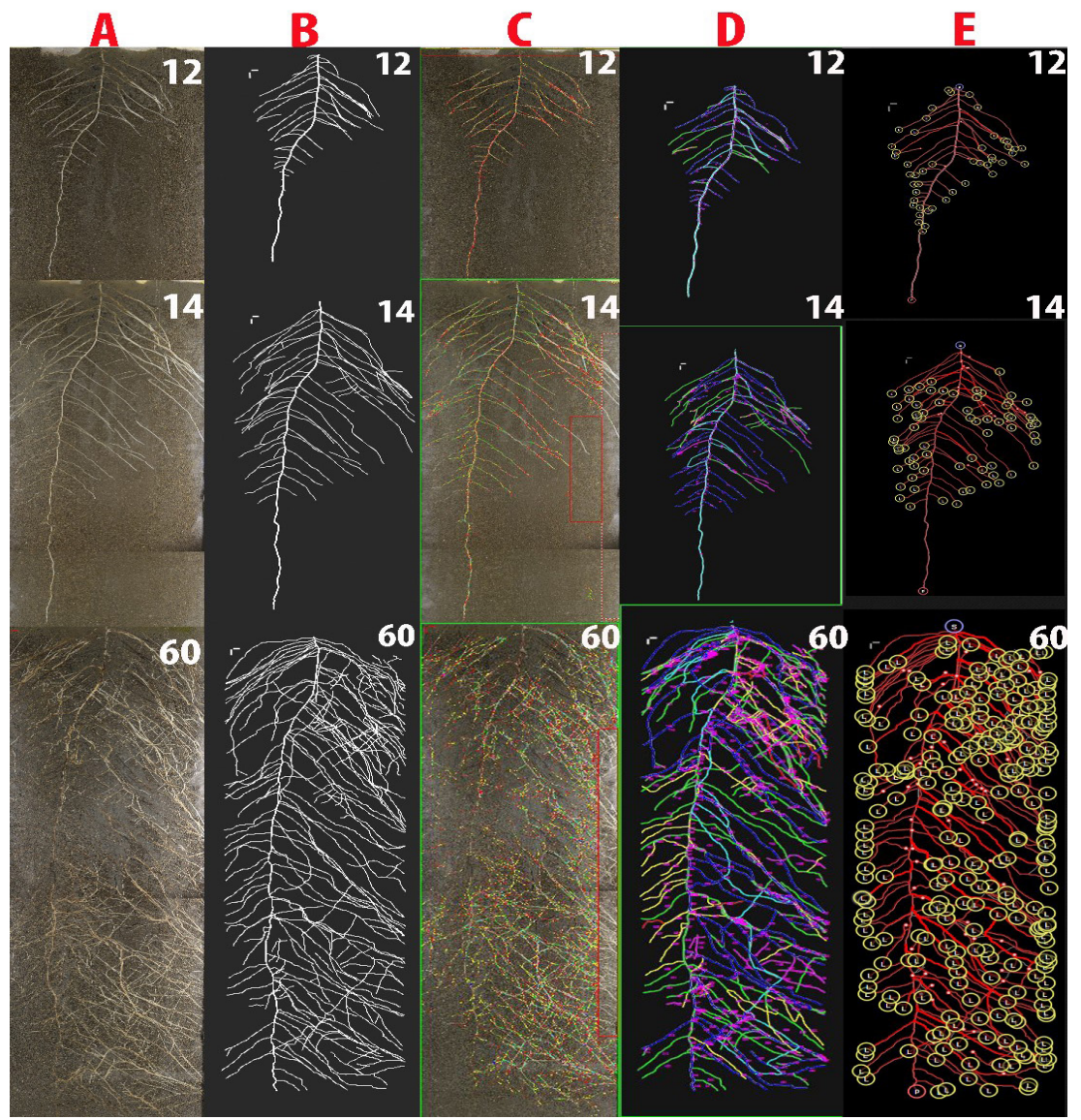

Fig. 1. Processing and analysis of faba bean rhizobox images taken at developmental stages 12,14 and 60 . A - original RGB images, $\mathrm{B}$ - binary images from manual tracking of the RGB original using CorelDraw X7, C - colour-based classification of root axes using WinRHIZO, D - WinRHIZO root analysis of manually tracked binary images, and E - RootNav analysis of manually tracked binary images.

Table 1. Root parameters derived from different image types (binary: manually tracked binary images: RGB: unprocessed RGB images; Scan: roots washed free from soil and images from roots scanned with a flatbed scanner) that are available from image analysis using the software WinRhizo and RootNav, respectively

\begin{tabular}{lc}
\hline Parameters & Software \\
\hline Root length $(\mathrm{cm})$ & Binary, RGB, Scan \\
& WinRhizo, RootNav \\
Bull area $\left(\mathrm{cm}^{2}\right)$ & RootNav \\
Number of lateral roots $(N)$ & WinRhizo, RootNav \\
Average emergence angle $\left(^{\circ}\right)$ & WinRhizo, RootNav \\
Magnitude $(N)$ & WinRhizo \\
External path length $(N)$ & WinRhizo \\
\hline
\end{tabular}

A statistical data analysis was performed using the PC program SAS version 9.4 (SAS Institute, Cary, USA). The root dataset was first evaluated through the use of analysis of variance (PROC GLM) in order to determine the effect of the factor "software" for common root traits that were quantified by using different analytical strategies (WinRhizo $_{\text {manual }}$ : WinRhizo with manual tracking on binary images $v s$. WinRhizo ${ }_{\text {auto }}$ : WinRhizo with automatic tracking on RGB images $v s$. RootNav: RootNav with manual tracking on binary images). PROC GLM was also used to reveal the effect of the growth stage on the individual traits. In order to perform an analysis of the usability of RGB raw images without pre-processing to estimate the root length as well as the relationship between the visible surface (imaging) $v s$. the total (scanned) root length, regression analysis (PROC REG) was used. The relationship between root traits (at different developmental stages) was assessed via Pearson correlation analysis (PROC CORR) and visualization through the use of a heat map. All traits were previously checked for normality (Kolmogorov-Smirnov test in PROC UNIVARIATE).

In order to highlight the direct and indirect pathways relating to the visible root imaging/phenotyping traits with their functional role in plant water uptake (transpiration), a structural equation model (SEM) was designed using PROC CALIS. It was hypothesized that root functionality (transpiration, obtained from changes in rhizobox weight) as an overall target, e.g. for stress resistance breeding, is driven by the overall extent of the root system (i.e. total living root length, living roots based on bright colour). The 
extent of the root system is driven by size related (hull area, external path length) and branching related traits (lateral number, angle, magnitude) as obtained from root phenotyping/imaging. SEM models with different possible paths connecting the variables (including the paths between root traits measured at vegetative and generative stages) were fit to the dataset. Among these models, the best one was identified using a set of statistical indicators. The recommendations of Schermelleh-Engel et al. (2003) were followed to combine different indicators (in this case: Standardized Root Mean Square Residual, Goodness-of-Fit Index, Bentler Bonett Normed and Nonnormed Fit Index) in order to reliably assess model fit and identify the best performing model to relate root phenotyping traits with plant functionality.

\section{RESULTS}

The root parameters measured at three developmental stages with WinRHIZO and RootNav differed significantly (Table 2). The traits obtained from manually tracked binary images were the lateral root number, emergence angle, hull area (RootNav) and lateral number, emergence angle, magnitude and external path length (WinRHIZO). The length was determined using binary and RGB images as well as washed/scanned roots at the end of the experiment (BBCH 60).

Overall, the two vegetative growth stages did not differ significantly (and were thus averaged for subsequent analysis), while all parameters except the root angle changed to a significant extent between the vegetative and flowering growth stages. In addition, those parameters obtained with both sets of software (lateral root number, angle of emergence, root length) revealed the differences in their segmentation/measurement approaches for the architectural description in particular (lateral root number, emergence angle) and at later for the developmental stages (BBCH 60).

With regard to root length, that was determined using both manually tracked binary and RGB raw images, the values obtained from colour-segmented images did not differ significantly from the values of binary images at vegetative stages, while at the flowering stage the differences were significant. Differences in root length estimates between manually tracked images using either WinRhizo or RootNav were greater than the differences between automatically segmented RGB images.

Table 2. Parameters from a root dataset of faba bean (three cultivars, two watering regimes) at vegetative $(\mathrm{BBCH} 12,14)$ and flowering (BBCH 60) growth stages quantified by different image analysis approaches: RootNav: manually tracked binary images evaluated

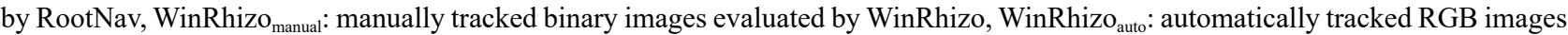
and washed/scanned roots evaluated by WinRhizo. Cumulative transpiration is included as a functional trait related to differences in the root systems. Different lowercase letters indicate significant differences between the growth stages for the single traits, different uppercase letters indicate significant differences between software for the common traits (Tukey HSD with $\mathrm{p}<0.05$, $\mathrm{n}=20$, mean (standard error)). See text for details

\begin{tabular}{|c|c|c|c|}
\hline Parameter & BBCH 12 & BBCH 14 & BBCH 60 \\
\hline \multicolumn{4}{|c|}{ RootNav } \\
\hline Root length (cm) & $345.0(56.0) \mathrm{aA}$ & $598.1(102.6) \mathrm{aA}$ & $3574.3(238.3) \mathrm{bA}$ \\
\hline Lateral number $(N)$ & $46.0(5.7) \mathrm{aA}$ & $65.5(7.4) \mathrm{aA}$ & $297.8(20.6) \mathrm{bA}$ \\
\hline Emergence angle $\left({ }^{\circ}\right)$ & $70.8(1.8) \mathrm{aA}$ & $67.3(1.1) \mathrm{aA}$ & $65.0(1.6) \mathrm{aA}$ \\
\hline Hull area $\left(\mathrm{cm}^{2}\right)$ & $377.7(51.7) \mathrm{a}$ & $611.0(59.5) \mathrm{a}$ & $2436.2(131.5) b$ \\
\hline \multicolumn{4}{|c|}{ WinRhizo $_{\text {manual }}$} \\
\hline Root length $(\mathrm{cm})$ & $225.2(31.0) \mathrm{aA}$ & $352.9(39.4) \mathrm{aA}$ & $1879.2(98.6) \mathrm{bB}$ \\
\hline Lateral number $(N)$ & $8.7(1.1) \mathrm{aA}$ & $7.8(1.3) \mathrm{aB}$ & $52.2(6.9) \mathrm{bB}$ \\
\hline Emergence angle $\left(^{\circ}\right)$ & $51.0(1.2) \mathrm{aA}$ & $51.4(1.0) \mathrm{aB}$ & $54.6(0.6) \mathrm{aB}$ \\
\hline External path length $(N)$ & $547.7(94.4) \mathrm{a}$ & $888.2(129.8) \mathrm{a}$ & $6617.7(1086.0) b$ \\
\hline Magnitude $(N)$ & $60.4(6.7) \mathrm{a}$ & $85.1(10.3) \mathrm{a}$ & $320.4(19.1) b$ \\
\hline \multicolumn{4}{|c|}{ WinRhizo $_{\text {auto }}$} \\
\hline Root length $_{\mathrm{RGB}}(\mathrm{cm})$ & $237.0(41.5) \mathrm{aA}$ & $388.4(62.1) \mathrm{aA}$ & $2542.9(240.8) b C$ \\
\hline Root length $_{\text {scan }}(\mathrm{cm})$ & - & - & 9897.9 (797.1) D \\
\hline Cumulative transpiration $(\mathrm{ml})$ & & & $597.3(292.6)$ \\
\hline
\end{tabular}


Figure 2 shows the regression of root length as determined from manually tracked binary images with reference to automatically segmented RGB images at the vegetative and flowering stages.

In all cases (vegetative and flowering stages, both sets of image analysis software), there was a significant relationship between the manually and automatically tracked root length. Using WinRHIZO, i.e. the same length measurement approach for both manual tracking and automatic colour segmentation respectively, an overestimation of root length from automatic RGB image segmentation may be observed for both growth stages ( slope $_{\text {vegetative }}=1.12$; slope $_{\text {flowering }}=1.99$ ). Comparing RootNav-measured binary images with RGB automatic segmentation in WinRHIZO, the length values obtained with RootNav were higher, particularly at the vegetative stage. The $\mathrm{R}^{2}$-values between the RootNav vs. RGB length estimates at both stages were lower $\left(\mathrm{R}_{\text {vegetative }}^{2}=0.84, \mathrm{R}_{\text {generative }}^{2}=0.57\right)$ compared to the values obtained when measuring both image types with WinRHIZO $\left(\mathrm{R}_{\text {vegetative }}^{2}=0.88, \mathrm{R}_{\text {generative }}^{2}=0.71\right)$.

The surface-visible roots of rhizobox phenotyping platforms represent a certain fraction of the entire root system. Inference concerning the plant root system based on rhizobox images thus assumes a the existence of a significant relationship between the visible root axes and the total root system. The prediction of mature root systems from early stage phenotyping would furthermore require a significant relationship between phenological growth stages.

On average, the visible root fraction at the flowering stage - when the rhizoboxes were emptied and the total root length was measured after washing and scanning - constituted about $27.7 \pm 11.1 \%$ of the total root length (depending on the image analysis method used). Image analysis derived root lengths at the vegetative stage were $12.2 \pm 7.1 \%$ of the flowering stage root length.

The relationship between surface-visible (vegetative and flowering stage) and scanned total root length is shown in Fig. 3. The results demonstrate that, independent of the root analysis software and image type (binary and RGB), the relationship between the visible and total root length is significant at the same growth stage (flowering), with the highest $\mathrm{R}^{2}$ value obtained for the colour-based WinRHIZO root length determination. Inference concerning total root length from the early, vegetative stage at flowering, however, was not feasible.

Analysing the inter-trait relationship between root system parameters may indicate a link between "higher-level" root traits such as root length or hull area and underlying traits such as the lateral number or emergence angle. The results of a correlation analysis between the measured parameters (Table 2) are shown in Fig. 4.

Generally, a more significant relationship between measured traits was found at the vegetative growth stage (31) as opposed to the flowering stage (18). The only parameters with values at the vegetative stage that scaled up towards the flowering stage was the lateral root number determined by WinRHIZO. The number of lateral roots at the vegetative stage was also related to the flowering stage root length.

For one particular set of software, the RootNav-derived parameters of root length and lateral root number were significantly correlated at both stages, and with the hull area at the vegetative stage only. WinRHIZO descriptors of root length, number of axes and magnitude correlate significantly at both stages, while angle, axis number, length and magnitude are only related at the vegetative stage.

Several root traits were highly correlated between the two image analysis programmes. Examples where there were significant correlations at both developmental stages highlight the consistent relationship between root traits derived from the two sets of software are: root length, number of axes and magnitude (WinRHIZO) with root length and lateral number (RootNav), and root length (WinRHIZO) with hull area and emergence angle (RootNav). Root angles originating from either set of software were either negatively (vegetative stage) or not (flowering stage) correlated. a

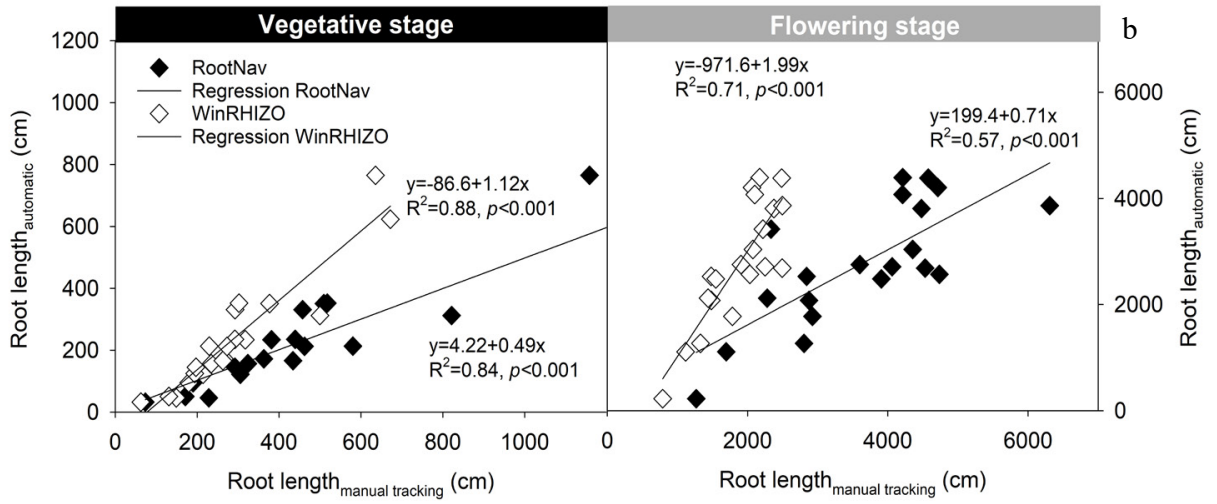

Fig. 2. Regression relationship of faba bean root length from manually tracked rhizobox images determined using RootNav and WinRHIZO, and automatically segmented RGB-images determined using WinRHIZO at the: $a$ - vegetative and $b$ - flowering growth stage. 


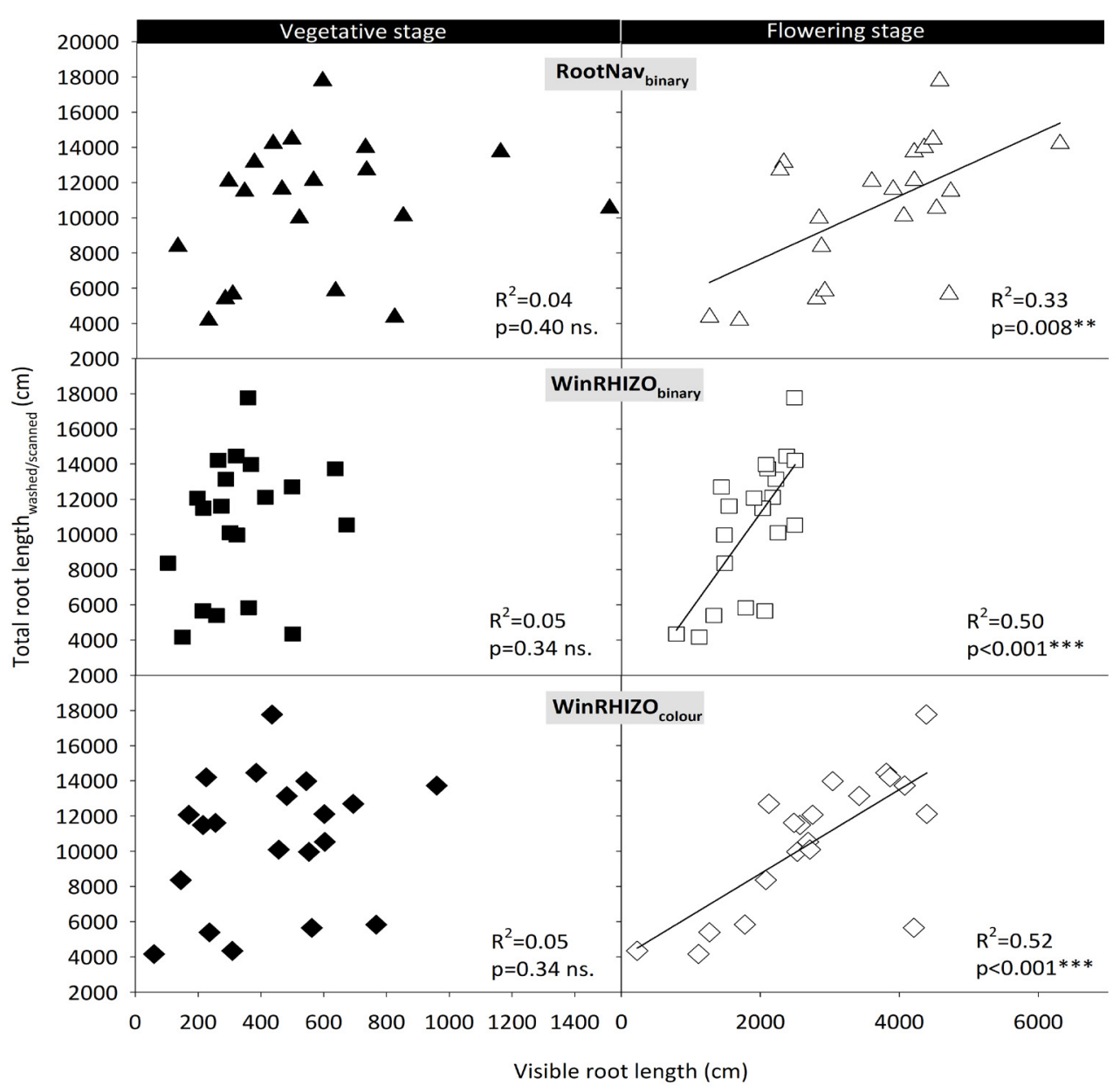

Fig. 3. Relationship of total root length determined after harvest (software: WinRHIZO) with the visible root length phenotyped at an early growth stage (vegetative) and at the same growth stage (flowering) - quantified using manually tracked binary (software: RootNav, WinRHIZO) and automatically segmented RGB-images (software: WinRHIZO).

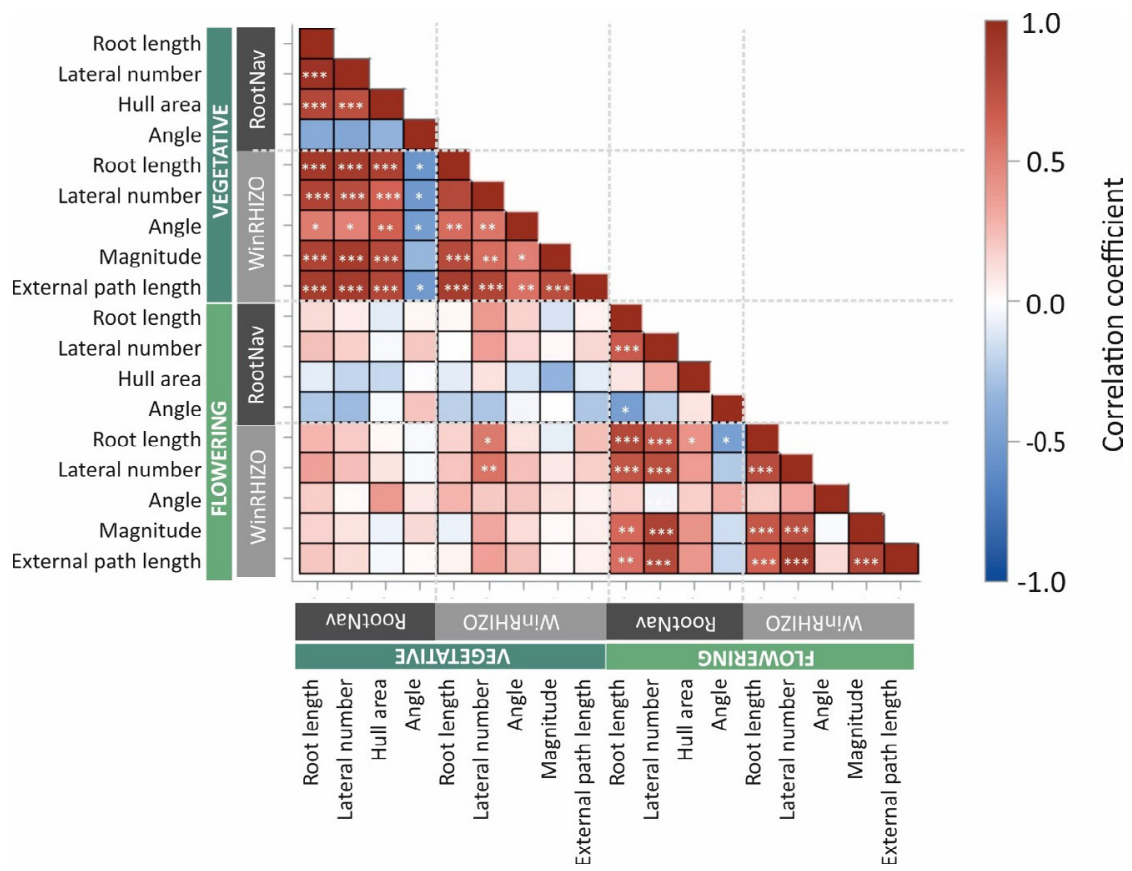

Fig. 4. Heat map showing the relationship (Pearson correlation coefficients) between faba bean root system traits at two principal growth stages (vegetative, flowering) using WinRHIZO and $\operatorname{RootNav}\left({ }^{*} \mathrm{p}<0.05, * * \mathrm{p}<0.01, * * * \mathrm{p}<0.001\right)$. 
Path analysis provides an approach to reveal causal pathways between the parameters in a dataset. In this case, the application of path analysis mainly aimed to 1) unravel key traits captured by rhizobox imaging that underlie total root length (from washed/scanned sampling after final imaging) and 2) relate traits that constitute a root system to plant transpiration as a key functional descriptor in the context of root studies. Figure 5 shows the resulting path model obtained from the dataset.

The path model links the vegetative stage with the flowering stage root system characterization, which in turn underlies the total root length (i.e. including the non-visible part during rhizobox imaging) and thereby drives root water uptake (transpiration).

The model is composed of determined root imaging parameters (from WinRHIZO and RootNav) that are considered to be lower-level constitutive traits (lateral root number, hull area, magnitude, external path length). The determined (manifest) parameters are grouped into two types of latent variables, i.e. branching and size descriptors. The magnitude (WinRHIZO) and lateral root number (RootNav) were considered to shape branching, while size was related to the external path length (WinRHIZO) and hull area (RootNav). Among the branching parameters, the root angle (from both sets of software) did not show any significant relationship with the branching descriptor (data not shown) and was therefore omitted from the final model. The final (exogeneous) predictor variable is cumulative plant transpiration. Furthermore, the branching descriptor at the vegetative stage did not show any significant pathways relating it to the other model components (data not shown). Therefore, this part of the model was excluded. In contrast, the vegetative growth stage size descriptor is linked to a significant extent to the flowering stage size descriptor. The two latent variables at the flowering stage (i.e. composite branching and size descriptors) are both related to each other to a significant extent and contributed to the factors resulting in total root length. Total root length finally relates to the exogenous variable of cumulative transpiration. In spite of its complexity, particularly through the inclusion of a vegetative-to-flowering stage relationship, the model had a satisfactory overall fit ( $c f$. model fit indices in Fig. 5).

In general, the direct links between the manifest variables and the latent composite descriptors were more significant as compared to the paths linking the single model components (vegetative stage size $\rightarrow$ flowering stage size $\leftrightarrow$ flowering stage branching $\rightarrow$ total root length $\rightarrow$ transpiration). Overall, the model reveals how the size and branching-related traits of the root system, as measured through image-based root phenotyping, scale towards the functionality of the root system for plant water uptake (transpiration).
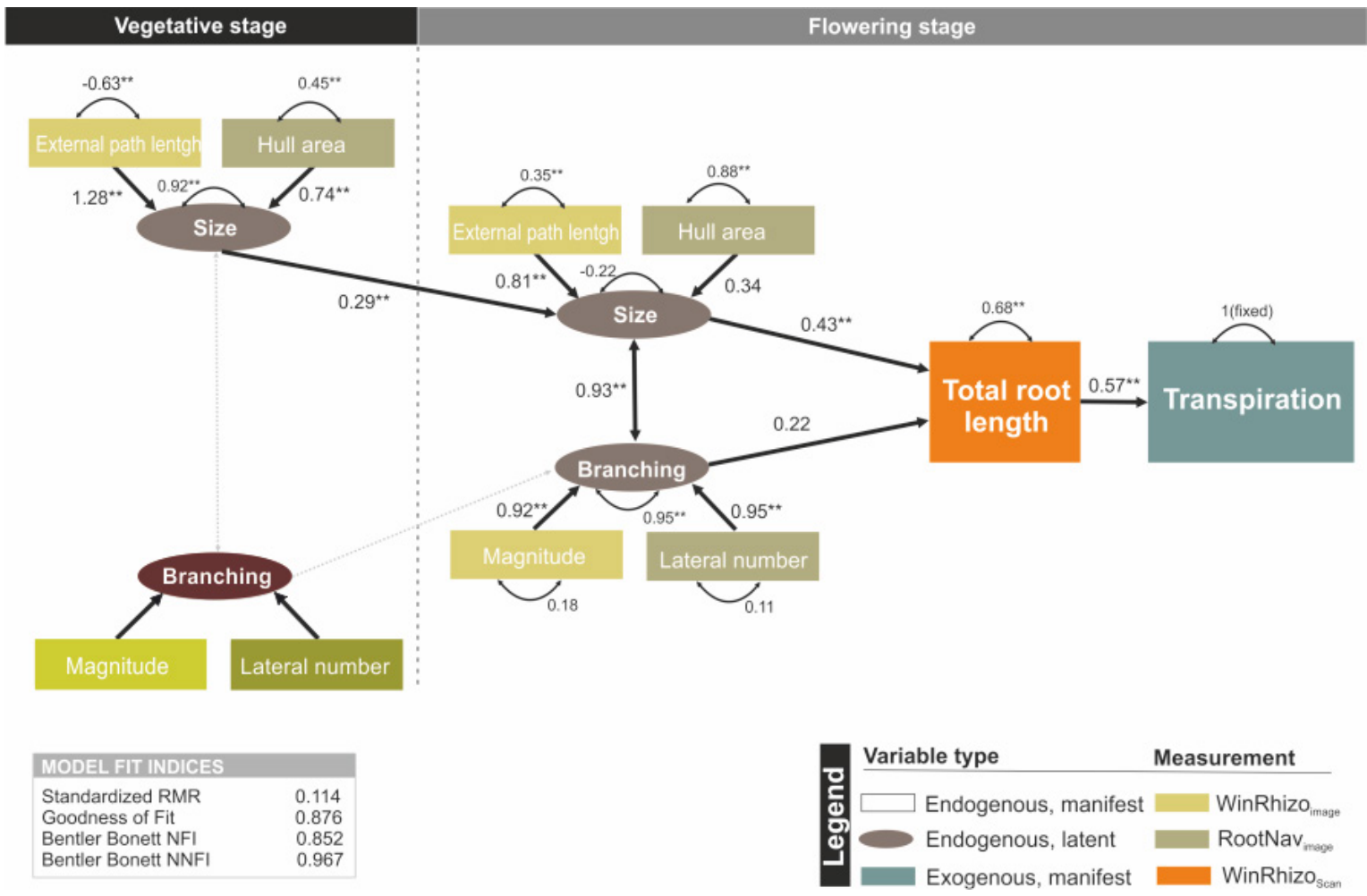

Fig. 5. Path model of single root imaging traits (grouped according to size and branching-related traits) and their relationship with: 1) total root length (washed/scanned) in terms of comprehensive high-level root system characteristics and 2) plant transpiration as a key target variable of functional root phenotyping. Numbers along straight arrows show the standardized path coefficients, while numbers on curved arrows show standardized variances $\left({ }^{*} \mathrm{p}<0.05, * * \mathrm{p}<0.01\right)$. 


\section{DISCUSSION}

With the advance in root phenotyping applications, analysis and trait extraction from images has become a major bottleneck in the process. This is particularly the case with rhizobox-based phenotyping platforms, the analysis of comparatively complex images of mature root systems, where only part of the entire root system is visible, prevents the wider application of these semi-natural systems. For length measurement, partial visibility (in this study $26.2 \%$ of the entire root length; for comparison: $32.4 \%$ in the faba bean rhizobox experiment of Belachew et al. (2018)) may be considered less problematic. In general, there is a significant relationship between visible and total length in rhizobox experiments (in this case $\mathrm{R}^{2}=0.52$, $\mathrm{p}<0.001$ for colour-segmented visible length and total length at the flowering stage; in comparison: $\mathrm{R}^{2}=0.76-0.79$ for chickpea and a slightly thinner $6 \mathrm{~mm}$ rhizobox system used by Bontpart et al., 2020). Thus, rhizobox phenotyping systems can be reliably used to make quantitative comparisons between root systems for different purposes such as genotype ranking in root breeding studies. For the soilbase systems, however, an accurate segmentation between the foreground (root) and background (soil) without the need for manual tracking would substantially increase the throughput of the technique and the range of practical applicability.

The software used in this study for root analysis (WinRhizo and RootNav) differed with regard to the automatic segmentation algorithms used (WinRhizo: thresholding, RootNav: expectation maximization classification) and the semi-automatic root tracking applied according to the different root orders (WinRhizo: multi-criterial (e.g. diameter, link numbers) pre-selection + manual post-processing; RootNav: low-cost path detection between base and tip + manual post-processing). Our results showed that concerning root length there was a significant correlation between the RootNav- and WinRhizo-derived data sets, i.e. both segmentation and tracking approaches provided similar results. With the increasing complexity of the root systems (between the vegetative and flowering stages), root length prediction from automatic segmentation algorithms, however, increasingly deviates from manually tracked images. This is probably related to a higher degree of root overlap and root axes growing in close proximity to each other, thereby introducing further bias in length measurement (Kimura et al., 1999; Himmelbauer et al., 2004).

However, when aiming to dissect root systems into their constitutive architectural traits (mainly branching frequency, emergence angle and the bending of lateral roots) image analysis requires the continuity of the single root axis from base to tip to be tracked (Leitner et al., 2014). Currently there is no software solution available for the automatic gap closure of root axes partially hidden in the substrate as is the case in rhizobox phenotyping systems. Recently Chen et al.
(2019) proposed the application of a neural network based algorithm to improve gap closure in different imaging tasks involving partially hidden thin structures. In addition, tracking complex (large size, frequently branching) root systems becomes increasingly uncertain and error-prone with a high number of overlapping axes (Kimura et al., 1999).

Therefore, the acquisition of architectural traits from rhizobox images (the number of lateral roots of a different order and their branching angles) still largely depends on manual tracking. On average, the time required to track a flowering-stage root image ( $c f$. Fig. $1 \mathrm{~B}, \mathrm{BBCH} 60$ ) is 50 to $70 \mathrm{~min}$. Even for the manually post-processed, gapclosed binary images single axis tracking by the software required further user-correction in the case of complex root systems with a high degree of overlap, further reducing throughput and the precision of image analysis. Thus, image pre-processing and correction for in-depth root architectural analysis still constitutes the main throughput constraint when aiming for a comprehensive architectural description of mature root systems grown in soil-based phenotyping systems.

However, dissecting global root system descriptors (root length) into their constitutive components which determine shape and structure is considered crucial to advancing the cause of targeted root management (via breeding or agronomic practices) as constitutive root architectural traits are less variable (lower genotype $\mathrm{x}$ environmental interaction) as compared to more adaptive global descriptors (Collins et al., 2008). Hodge et al. (2009) categorized root architectural descriptors into two types: shape-parameters related to the spatial location of roots and soil volume foraging (angle of emergence, hull area), and also structural parameters which are defined as constitutive building units (lateral root number). The image analysis tools applied in this study provided common architectural descriptors (lateral root number), while others were based on different concepts (hull area in RootNav; topological traits in WinRhizo based on Fitter, 1987).

Lateral root number correlated to a significant extent between the two software packages at both the vegetative and flowering stages. The software-specific descriptors (hull area, magnitude, external path length) showed significant relationships with the lateral root number and root length, thus providing a link between the branching pattern and size.

Different outcomes between RootNav and WinRhizo were found for root angle, with an absence of or even slightly negative (in the case of the vegetative stage) correlations. We consider that quantifying the emergence angle of laterals from parent root axes as a descriptor of root system shape is challenging as it strongly depends on fitting a straight line (tangent) to a bending root axes. The coefficient of variation in root angles was lowest among all of the measured root parameters (between 5.9 and 10.2\%). This may indicate a lack of sensitivity in angle measurement 
(but also low genotypic and/or environmental effects, which, however, is unlikely considering the results of studies concerning variability in root angle among genotypes and the response to nutrients (Trachsel et al., 2013).

Hull area provides an alternative descriptor to root angle which also captures the space occupation of the root system. Several studies have shown hull area to be related to a significant extent to root length and rooting depth (Bodner et al., 2019), which was also confirmed in this study. At the vegetative stage, the number of lateral roots was essentially shaping the hull area. At the flowering stage, however, the occupation of space described by the hull area is beyond the number of lateral roots, becoming a more complex function of elongation and the bending of the single root axes.

Overall, the inter-relation among root traits demonstrates that the key architectural trait shaping the automatically captured composite traits of root length and hull area is the lateral root number. While the spacing of lateral root primordia along a parent axes has been reported as a genetically constitutive trait, the emergence of the lateral root interacts with the soil environment (water, P, N availability; Dubrovsky et al., 2009). Creating more advanced (semi-automated) image analysis tools for determining the lateral root number based on root-based phenotyping systems (extracting visible axes with a minimum length from the whole root system image and counting their lateral numbers in order to obtain a representative average estimate) therefore has a high degree of significance for root system breeding for the purpose of improving adaptation to resource limiting environments.

The functional relationship between single root traits and root functionality (root water and/or nutrient uptake) is complex, as it not only involves root architecture but also physiology and anatomy (root membrane transporters, root xylem hydraulics; Vadez, 2014; Freschet et al., 2021). In addition, the overall allocation pattern of assimilates between shoot and root is closely interrelated with resource availability and extraction (Foxx and Fort, 2019). We therefore suggest the use of path models as an empirical approach in order to reveal the links between single constitutive root traits (as potential breeding targets), global root descriptors (mainly root length as a trait measurable in the field, e.g. for validation purposes) and the targeted functions at plant level such as transpiration (measured by weighing in this case) or nutrient uptake. There was a weak, but still significant relationship between the vegetative and flowering stage root size, but not with the branching structure. Thus, for faba bean, upscaling from the early vegetative root screens to mature root size seems feasible (in contrast to monocots; Watt et al., 2013), although overcoming linear scaling might also still be advantageous for dicots (Zhao et al., 2017). The path model highlighted that the latent variables describing the size and branching pattern (and integrating the single constitutive imaging traits) were closely inter-dependent, while the link/path between total root length and plant water supply (transpiration) was mainly driven by the size descriptor.

In considering this trait-function link and the above-described throughput challenges to obtain architectural traits from rhizobox images (manual gap closure), we suggest that rhizobox datasets should focus on visible root length and hull area which provide robust descriptors with meaningful (empirical) relationships to root functionality (transpiration). Rather than compromising on throughput (one of the aims of modern phenotyping) with a specific phenotyping approach (such as rhizoboxes) by using a laborious manual image post-processing, data links across phenotyping systems should be studied. In this way, the advantage of realistic growth conditions (substrate and growth duration) in rhizoboxes could be combined with certain architectural details from approaches specifically targeting these traits (germination paper seeding root screens, Gioia et al., 2017). Also, for this purpose, path analysis might provide an appropriate empirical approach to highlight such cross-platform relationships. In line with this course of action, each phenotyping system has its specific parameter set and thereby defines the image analysis software (or combination of software) to be used. Overall, the application of SEM could better connect datasets from different (phenotyping system-specific) environments and between (software-specific) root traits, thereby providing a successively more comprehensive picture concerning root system characteristics and functionality.

\section{CONCLUSIONS}

1. Imaging the visible length of root axes on the surface of soil-filled rhizoboxes as a semi-natural phenotyping system and using automated analysis of the obtained RGB images provides a reliable estimate of total root length. Different segmentation approaches between roots and soil provide similar results, while inference from early developmental stages towards mature root length is not possible.

2. The measurement of root architecture traits requires the manual post-processing of images to close the gaps of the visible root axes. Different types of architectural traits provided by software tools are inter-comparable.

3. Combining root size and branching descriptors obtained from rhizobox phenotyping can be used to predict plant functionality (transpiration). Structural equation modelling can be used to reveal which traits are essential to obtain root phenotyping datasets to infer which plants would have an effective uptake of soil resources.

4. The automatization of image processing, with particular reference to gap closure and single axis tracking of crossing roots, is essential to enhance the throughput of rhizobox phenotyping systems for providing root architecture datasets obtained under semi-natural growing conditions. 


\section{ACKNOWLEDGMENTS}

We kindly thank the EUROLEGUME donors for the genetic material. Ina Alsiņa, Latvia and Aleš Látr, Czech Republic kindly provided the rhizobia and mycorrhizal inoculum, respectively.

Conflict of interest: The authors declare no conflict of interest.

\section{REFERENCES}

Belachew K.Y., Nagel K.A., Fiorani F., and Stoddard F.L., 2018. Diversity in root growth responses to moisture deficit in young faba bean (Vicia faba L.) plants. PeerJ 6, e4401. https://doi.org/10.7717/peerj.4401

Bodner G., Alsalem M., Nakhforoosh A., Arnold T., and Leitner D., 2017. RGB and spectral root imaging for plant phenotyping and physiological research: Experimental setup and imaging protocols. JoVE (J. Visualized Experiments), e56251. https://doi.org/10.3791/56251

Bodner G., Loiskandl W., Hartl W., Erhart E., and Sobotik M., 2019. Characterization of cover crop rooting types from integration of rhizobox imaging and root atlas information. Plants, 8, 514. https://doi.org/10.3390/plants8110514

Bodner G., Nakhforoosh A., Arnold T., and Leitner D., 2018. Hyperspectral imaging: a novel approach for plant root phenotyping. Plant Methods, 14, 84.

https://doi.org/10.1186/s13007-018-0352-1

Bontpart T., Concha C., Giuffrida M.V., Robertson I., Admkie K., Degefu T., Girma N., Tesfaye K., Haileselassie T., and Fikre A., 2020. Affordable and robust phenotyping framework to analyse root system architecture of soilgrown plants. Plant J., 103, 2330-2343.

https://doi.org/10.1111/tpj.14877

Chen H., Valerio Giuffrida M., Doerner P., and Tsaftaris S.A., 2019. Adversarial large-scale root gap inpainting. Proc. IEEE/CVF Conf. Computer Vision and Pattern Recognition, pp. 0-0. https://doi.org/10.1109/CVPRW.2019.00318

Chen Y., Ghanem M.E., and Siddique K.H.M., 2017. Characterising root trait variability in chickpea (Cicer arietinum L.) germplasm. J. Exp. Botany, 68, 1987-1999. https://doi.org/10.1093/jxb/erw368

Chen Y., Rengel Z., Palta J., and Siddique K.H.M., 2018. Efficient root systems for enhancing tolerance of crops to water and phosphorus limitation. Indian J. Plant Physiol., 1-8. https://doi.org/10.1007/s40502-018-0415-3

Collins N.C., Tardieu F., and Tuberosa, R., 2008. Quantitative trait loci and crop performance under abiotic stress: where do we stand? Plant Physiology, 147, 469-486. https://doi.org/10.1104/pp.108.118117

Delory B.M., Weidlich E.W.A., Meder L., Lütje A., van Duijnen R., Weidlich R., and Temperton V.M., 2017. Accuracy and bias of methods used for root length measurements in functional root research. Methods Ecol. Evolution, 8, 1594-1606. https://doi.org/10.1111/2041-210X.12771

Downie H.F., Adu M.O., Schmidt S., Otten W., Dupuy L.X., White P.J., and Valentine T.A., 2015. Challenges and opportunities for quantifying roots and rhizosphere interactions through imaging and image analysis. Plant, Cell Environ., 38, 1213-1232. https://doi.org/10.1111/pce.12448

Dubrovsky J.G., Soukup A., Napsucialy-Mendivil S., Jeknić Z., and Ivanchenko M.G., 2009. The lateral root initiation index: an integrative measure of primordium formation. Annals Botany, 103, 807-817. https://doi.org/10.1093/aob/mcn267

Fitter A.H., 1987. An architectural approach to the comparative ecology of plant root systems. New Phytologist, 106, 61-77. https://doi.org/10.1111/j.1469-8137.1987.tb04683.x

Freschet G.T., Roumet C., Comas L.H., Weemstra M., Bengough A.G., Rewald B., Bardgett R.D., De Deyn G.B., Johnson D., and Klimešová J., 2021. Root traits as drivers of plant and ecosystem functioning: current understanding, pitfalls and future research needs. New Phytologist, 232, 1123-1158.

Foxx A.J. and Fort F., 2019. Root and shoot competition lead to contrasting competitive outcomes under water stress: A systematic review and meta-analysis. PloS one, 14, e0220674. https://doi.org/10.1371/journal.pone.0220674

Gioia T., Galinski A., Lenz H., Müller C., Lentz J., Heinz K., Briese C., Putz A., Fiorani F., and Watt M., 2017. GrowScreen-PaGe, a non-invasive, high-throughput phenotyping system based on germination paper to quantify crop phenotypic diversity and plasticity of root traits under varying nutrient supply. Functional Plant Biol., 44, 76-93. https://doi.org/10.1071/FP16128

Haling R.E., Yang Z., Shadwell N., Culvenor R.A., Stefanski A., Ryan M.H., Sandral G.A., Kidd D.R., Lambers H., and Simpson R.J., 2016. Root morphological traits that determine phosphorus-acquisition efficiency and critical external phosphorus requirement in pasture species. Functional Plant Biol., 43, 815-826. https://doi.org/10.1071/FP16037

Himmelbauer M.L., Loiskandl W., and Kastanek F., 2004. Estimating length, average diameter and surface area of roots using two different image analyses systems. Plant and Soil, 260, 111-120. https://doi.org/10.1023/B:PLSO.0000030171.28821.55

Hodge A., Berta G., Doussan C., Merchan F., and Crespi M., 2009. Plant root growth, architecture and function. Plant Soil, 321, 153-187. https://doi.org/10.1007/s11104-009-9929-9

Johnson M.G., Tingey D.T., Phillips D.L., and Storm M.J., 2001. Advancing fine root research with minirhizotrons. Environ. Exp. Botany, 45, 263-289. https://doi.org/10.1016/S0098-8472(01)00077-6

Kashiwagi J., Krishnamurthy L., Crouch J.H., and Serraj R., 2006. Variability of root length density and its contributions to seed yield in chickpea (Cicer arietinum L.) under terminal drought stress. Field Crops Res., 95, 171-181. https://doi.org/10.1016/j.fcr.2005.02.012

Kimura K., Kikuchi S., and Yamasaki Si., 1999. Accurate root length measurement by image analysis. Plant Soil, 216, 117-127. https://doi.org/10.1023/A:1004778925316

Leitner D., Felderer B., Vontobel P., and Schnepf A., 2014. Recovering root system traits using image analysis exemplified by two-dimensional neutron radiography images of lupine. Plant Physiol., 164, 24-35. https://doi.org/10.1104/pp.113.227892 
Lobet G., 2017. Image analysis in plant sciences: publish then perish. Trends Plant Sci., 22, 559-566. https://doi.org/10.1016/j.tplants.2017.05.002

Lynch J.P., 2011. Root phenes for enhanced soil exploration and phosphorus acquisition: tools for future crops. Plant Physiol., 156, 1041-1049. https://doi.org/10.1104/pp.111.175414

Manschadi A.M., Christopher J., and Hammer G.L., 2006. The role of root architectural traits in adaptation of wheat to water-limited environments. Functional Plant Biol., 33, 823-837. https://doi.org/10.1071/FP06055

Minervini M., Scharr H., and Tsaftaris S.A., 2015. Image analysis: the new bottleneck in plant phenotyping [applications corner]. IEEE Signal Processing Magazine, 32, 126-131. https://doi.org/10.1109/MSP.2015.2405111

Nagel K.A., Putz A., Gilmer F., Heinz K., Fischbach A., Pfeifer J., Faget M., Blossfeld S., Ernst M., and Dimaki C., 2012. GROWSCREEN-Rhizo is a novel phenotyping robot enabling simultaneous measurements of root and shoot growth for plants grown in soil-filled rhizotrons. Functional Plant Biol., 39, 891-904. https://doi.org/10.1071/FP12023

Pang J., Ryan M.H., Lambers H., and Siddique K.H.M., 2018. Phosphorus acquisition and utilisation in crop legumes under global change. Current Opinion Plant Biol., 45, 248254. https://doi.org/10.1016/j.pbi.2018.05.012

Pfeifer J., Faget M., Walter A., Blossfeld S., Fiorani F., Schurr U., and Nagel K.A., 2014. Spring barley shows dynamic compensatory root and shoot growth responses when exposed to localised soil compaction and fertilisation. Functional Plant Biol., 41, 581-597. https://doi.org/10.1071/FP13224

Pierret A., Gonkhamdee S., Jourdan C., and Maeght J.-L., 2013. IJ_Rhizo: an open-source software to measure scanned images of root samples. Plant Soil, 373, 531-539. https://doi.org/10.1007/s11104-013-1795-9
Pound M.P., French A.P., Atkinson J.A., Wells D.M., Bennett M.J., and Pridmore T., 2013. RootNav: navigating images of complex root architectures. Plant Physiol., 162, 18021814. https://doi.org/10.1104/pp.113.221531

Rose L. and Lobet G., 2019. Accuracy of image analysis tools for functional root traits: A comment on Delory et al. (2017). Methods Ecol. Evolution, 10(5), 702-711.

Schermelleh-Engel K., Moosbrugger H., and Müller H., 2003. Evaluating the fit of structural equation models: Tests of significance and descriptive goodness-of-fit measures. Methods of Psychological Research online, 8, 23-74.

Trachsel S., Kaeppler S.M., Brown K.M., and Lynch J.P., 2013. Maize root growth angles become steeper under low $\mathrm{N}$ conditions. Field Crops Res., 140, 18-31. https://doi.org/10.1016/j.fcr.2012.09.010

Vadez V., 2014. Root hydraulics: the forgotten side of roots in drought adaptation. Field Crops Res., 165, 15-24. https://doi.org/10.1016/j.fcr.2014.03.017

Wagner S., Hoefer C., Prohaska T., and Santner J., 2020. Twodimensional visualization and quantification of labile, inorganic plant nutrients and contaminants in soil. J. Vis. Exp., 163, e61661. https://doi.org/10.3791/61661

Wang T., Rostamza M., Song Z., Wang L., McNickle G., IyerPascuzzi A.S., Qiu Z., and Jin J., 2019. SegRoot: a high throughput segmentation method for root image analysis. Computers Electronics Agric., 162, 845-854. https://doi.org/10.1016/j.compag.2019.05.017

Watt M., Moosavi S., Cunningham S.C., Kirkegaard J.A., Rebetzke G.J., and Richards R.A., 2013. A rapid, controlled-environment seedling root screen for wheat correlates well with rooting depths at vegetative, but not reproductive, stages at two field sites. Annals Botany, 112, 447-455. https://doi.org/10.1093/aob/mct122

Zhao J., Bodner G., Rewald B., Leitner D., Nagel K.A., and Nakhforoosh A., 2017. Root architecture simulation improves the inference from seedling root phenotyping towards mature root systems. J. Exp. Botany, 68, 965-982. https://doi.org/10.1093/jxb/erw494 